\title{
Authors' response to Li et al. Obes Facts 2015;8:DOI: 10.159/000381434
}

\author{
Rosely Sichieri Diana Barbosa Cunha \\ Department of Epidemiology, Institute of Social Medicine, State University of \\ Rio de Janeiro, Rio de Janeiro, Brazil
}

Li et al. [1] have concerns regarding our statement that adjusting for the baseline values of parameters that are highly influenced by baseline values can bias the results in randomized studies. They argued that this is a standard procedure and that our study should be retracted mainly because it contradicts the epidemiology literature.

Our study cannot be retracted exactly because it indicates this possibility of bias. We are not arguing that randomization procedure with unbalanced baseline is due to chance, therefore there is no selection bias. However, in this situation, usually in school-based interventions to prevent obesity, adjustment may have biased the results.

We observed in our systematic review that $35 \%$ of the reviewed studies had statistically significant unbalanced measures of BMI at baseline. We also highlight that cluster designs favor unbalanced baseline measures.

The statement highly criticized in the letter by Li et al. [1] was demonstrated in our analysis of a cluster randomized trial, as indicated in the discussion of our paper [2]. This analysis showed no association between intervention and BMI change when analysis was uncontrolled for baseline BMI and changed to a statistically significant association when baseline BMI was included in the model.

Li et al. [1] demand that the article should be retracted based on two points that we will address further, but did not comment on this specific result [2] showing a biased conclusion after baseline adjustment. Also, they ignore our main suggestion that 'procedures that disentangle the effects of group, time and changes in time, such as mixed effects models, should be used as standard methods in school-based studies'. This kind of analysis clearly indicated that mean baseline BMI was the main difference between groups, without differences in the longitudinal change in our study [2]. Therefore, we have documented the possibility of bias by baseline adjustment for BMI in this specific study. In the review paper of school-based interventions [3], we evaluated the magnitude of unbalanced BMI between groups at baseline and how authors have analyzed their data.

Li et al. [1] concluded that our assumption is simply untrue, but we have shown i) the effect of baseline adjustment and ii) the high percentage of unbalanced BMI data (35\%) at baseline in the reviewed studies. 
With regard to Li's et al. [1] criticism of the references that we used to support our main research question, we agree that all of them are related to observational studies not applying experimental designs. There are no references related to experimental designs because, as the authors indicated in the letter, by design we expect lack of bias. However, we clearly stated after reporting the findings from observational studies that 'For experimental designs, the same principles apply with the additional challenge that data are expected to be balanced at baseline. The reviewed data show that cluster designs favor imbalance, unless the number of clusters is high'.

The criticism of Li et al. [1] about sample size is unfair since we are comment on the needed sample size for individually based studies. For any clustered study the sample size will be greater. We clearly stated 'because schools, not individual children, are randomized'. In most studies, sample size calculations were based on a change of approximately 1 BMI unit, which is too large for most primary prevention trials.

\section{Disclosure Statement}

The authors have read and approved this version of the manuscript. None of the authors have any conflicts of interest.

\section{References}

1 Li P, Brown AW, Dawson JA, Kaiser KA, Bohan Brown MM, Keith SW, Oakes JM, Allison DB: Concerning Sichieri R, Cunha DB: Obes Facts 2014;7:221-232. The assertion that controlling for baseline (pre-randomization) covariates in randomized controlled trials leads to bias is false. Obes Facts 2015;8:DOI: 10.159/000381434.

$\checkmark 2$ Cunha DB, de Souza BN, Pereira RA, Sichieri R: Effectiveness of a randomized school-based intervention involving families and teachers to prevent excessive weight gain in Brazil. PLoS One 2013;8:e57498.

-3 Sichieri R, Cunha DB: Unbalanced baseline in school-based interventions to prevent obesity: adjustment can lead to bias - a systematic review. Obes Facts 2014;7:221-232. 